\title{
Candidater et embaucher : le courrier de candidature comme dispositif de rencontre
}

\author{
Yves LOCHARD *, Pascal UGHETTO **
}

Les opérations d'embauche semblent soumises, ces dernières années, à une véritable révolution introduite par la propagation rapide de l'utilisation d'Internet tant par les candidats que par les recruteurs (Fondeur et Tuchszirer, 2005). On peut estimer que l'on assiste à la mise en place de supports de mise en relation, de pratiques et de représentations, qui constituent autant de nouveautés, de changements réels dans l'attente d'être répertoriés et interprétés. De ce point de vue, la numérisation qui se développe actuellement est l'occasion de nouvelles avancées dans l'analyse des phénomènes d'embauche, dans la compréhension des enjeux et stratégies se déployant dans ce domaine et il importe, comme en témoigne ce volume, que, pour la connaissance des processus d'embauche, les recherches se déploient sur ce nouveau contexte.

Les classiques lettre de motivation et $\mathrm{CV}$ constituent un dispositif de rencontre entre postulants et recruteurs que tout un chacun avait fini par considérer comme naturel et indépassable. En face des évolutions récentes, l'une et l'autre pourraient avoir tendance à être déclassés comme des vecteurs sans mystère et sans enjeux, et donc peu dignes de l'investigation économique ou sociologique. C'est pourtant la vertu de certains marchés du travail, comme celui des personnels de la grande distribution alimentaire, que de retenir l'observateur dans le basculement vers un intérêt exclusif pour le recrutement numérisé. Candidats et employeurs se retrouvant sur de tels marchés du travail vivent dans un univers où Internet n'est pas en train de bouleverser les supports de leur rencontre, n'est pas en passe de rendre su-

* Chercheur à I'IRES.

** Université de Marne-la-Vallée, LATTS. 
rannés la lettre de motivation et le $\mathrm{CV}$. Au contraire, dans cet environnement, ces derniers continuent à être perçus comme faisant problème, suscitant de la discussion, du débat : loin de les concevoir comme des outils neutres, exempts de pièges et d'embûches avant le grand saut vers internet, postulants et recruteurs continuent de s'y débattre avec une norme d'action et de représentation que beaucoup questionnent, implicitement ou explicitement.

A la lumière d'un tel environnement, on est également invité à prendre conscience que le recrutement par Internet tend, pour l'instant, à concerner de manière privilégiée des catégories de salariés très spécifiques, plutôt situés sur des segments avantageux de l'échelle des catégories socio-professionnelles et bien dotés en capital culturel. Le marché du travail de la grande distribution alimentaire, qui connait de structurels besoins de recrutement, concerne des personnels habituellement considérés comme «non ou peu qualifiés » (caissières, employés de libre-service) et, dans des volumes un peu plus restreints, des titulaires de postes de niveau maitrise, comme les chefs de rayon. Le directeur d'un supermarché n'est pas assimilé à l'un de ces cadres qu'un employeur aurait l'idée de chasser par Internet. Or, tous ces gens se figurent largement le marché du travail à partir de notions de proximité géographique (on pose sa candidature au supermarché où l'on va faire ses courses) et de filières de progression interne (hypermarchés et supermarchés mobilisent fortement la mobilité interne ascendante pour pourvoir les postes sur les différents niveaux hiérarchiques).

Aussi, à l'heure d'Internet, il devient urgent d'observer attentivement les problèmes que l'on se pose dans de tels environnements, les problèmes pratiques ou pragmatiques auxquels postulants et recruteurs font face pour se rencontrer et faire affaire. Le cas de la grande distribution alimentaire démontre qu'il n'est pas besoin de technologies très poussées comme Internet pour que les acteurs aient l'impression de devoir composer avec un dispositif technique, ou socio-technique, qui, à la fois, est là pour les équiper, pour les aider à se rencontrer, et leur pose problème, suscite chez eux des réactions, des interrogations, le sentiment parfois d'être encombrés et entravés.

Car rien ne serait plus faux que de penser qu'un marché du travail avant Internet ne serait peuplé que d'humains, dépourvus de la moindre technique. CV et lettre de motivation ne sont pas des objets rudimentaires, non concernés par la technicité. Ils sont, au contraire, profondément imprégnés de procédés, d'inventions visant à créer de l'efficacité, à rendre l'action efficace : action de postuler ou action de recruter. Tant le contenu que la forme sont soumis à des obligations, des normes, défendues par des professionnels s'exprimant dans des guides, des manuels, des titres de la presse spécialisée, et qui imprègnent ce faisant l'acte de candidature de techniques censées ai- 
der le postulant à atteindre son effet et le recruteur à trouver le bon individu. Ces techniques tendent à faire partie des évidences partagées par chacun dans le monde contemporain; au moment de faire acte de candidature ou de sélectionner des candidats, elles n'en sont pas moins profondément ressenties comme des corps, des objets, auxquels il faut s'habituer, qu'il faut apprendre à manipuler. Comme tout dispositif socio-technique (pour une présentation, voir Peeters et Charlier, 1999), ceux qui en font l'expérience éprouvent à la fois la propriété qui est la leur de démultiplier les capacités d'action et leur caractère contraignant où s'instillent les catégories de pensée des concepteurs. Si être un candidat postulant par le biais d'internet n'a rien de spontané et si, le temps que la familiarité avec l'outil se crée, on peut se sentir plus encombré qu'aidé par l'outil (Beauvallet et al., ce numéro), il n'en va guère autrement pour certains rédacteurs de courriers de candidature qui auraient plus spontanément imaginé de se déplacer au magasin et de dire, par exemple, qu'ils connaissaient bien tel(le) ami(e) qui y est déjà employé(e).

Un double matériel empirique nous servira ici de support d'analyse (Lochard et Ughetto, 2004 et 2006) : d'un côté, un corpus de courriers de candidature envoyés à la DRH des hypermarchés France d'un groupe de la grande distribution (une centaine de candidatures spontanées et en réponse à des annonces, sur des postes d'employés et de cadres); de l'autre, une série d'entretiens avec des directeurs de supermarchés de ce groupe (questionnés sur les principes qu'ils se fixent pour recruter, les contraintes qu'ils y voient, les attentes à l'égard des candidats, les outils utilisés, l'usage du CV et de la lettre, la conduite de l'entretien...) ainsi que diverses observations (limitées, dans cet article, à une séance d'entretiens de prérecrutement de futurs chefs de rayons et directeurs de magasins).

L'analyse a été conduite en cherchant à appréhender postulants et recruteurs comme confrontés à des problèmes pratiques ou pragmatiques, occupés par le souci de ce qui peut les aider à se rendre efficaces dans les efforts qu'ils accomplissent, les visées qui sont les leurs, et d'abord en faisant face avant tout au problème de la distance qui les sépare de celui qu'ils recherchent. Emmanuelle Marchal et Didier Torny (2003) ont fort opportunément rappelé, en étudiant les annonces d'offre d'emploi, que celles-ci ont commencé par accompagner la démarche consistant pour le postulant à se présenter directement chez l'employeur et ont fini par s'y substituer, ce qui fait naître toute une série d'enjeux autour du CV et de la lettre : aux fins de sélectionner et de préparer l'entretien avec un petit nombre de candidats, ceux-ci doivent concentrer, indépendamment de tout contact, de l'information qui se révélait auparavant dans l'interaction physique (permettant de jauger la personne) et la discussion. 
En observant postulants et recruteurs autour de la médiation de leur rencontre que constitue l'écrit, on voit in concreto le double problème d'action que les innovations autour d'Internet renouvellent plus qu'abolissent. De fait, ce double problème se révèle plus structurel que simplement lié à la numérisation : il s'agit, d'une part, de la "marge intensive » et non pas seulement extensive de l'information (Rees, 1966) sur laquelle raisonne le recruteur (et que le candidat tente de travailler), soit le fait que, à partir d'un certain stade, l'utilité n'est pas retirée de l'ajout d'une offre de travail supplémentaire mais de l'identification de la qualité différentielle du candidat ; d'autre part, il s'agit de l'information peu objectivable (l'information à forte bande passante évoquée par Autor, 2001), notamment tout ce qui concerne les «motivations ».

\section{L'embauche sous l'exigence d'une professionnalisation des candidats et des recruteurs}

Même lorsqu'on se fait recommander, lorsqu'on passe par les réseaux sociaux (Granovetter, 1974), même pour un job d'été, postuler à un emploi passe désormais presque toujours par l'envoi d'un CV et d'une lettre de motivation. Ces composantes de base du dispositif organisant la rencontre avec l'employeur potentiel ne se manient pas sans que les candidats y soient formés (manuels, conseils des intermédiaires du marché du travail...) ni sans un apprentissage sur les techniques de décryptage du côté des recruteurs. La professionnalisation du recrutement impose celle des candidats (Lochard et Ughetto, 2004) et des recruteurs.

\section{L'obligation sociale de la professionnalisation de l'embauche...}

Un courrier de candidature ne se rédige pas dans une absolue liberté, quoiqu'il soit censé être l'expression du candidat, de sa singularité. Guides du bon candidat, magazines, conseils divers sont là pour lui rappeler les normes du genre et que, à l'ère des ressources humaines, l'embauche est supposée ne plus se satisfaire d'aucun amateurisme, immédiatement assimilé à de la négligence envers l'employeur, à un manque d'enthousiasme pour l'emploi convoité. Postulants et recruteurs sont ainsi invités à appréhender les écrits de candidature, non comme des outils de mise en relation purement fonctionnels, mais comme des documents par lesquels le candidat se dit, c'est-à-dire non seulement se déclare mais dit ce qu'il est et ce qu'il sera dans son travail. L'écrit est ainsi la base d'un dispositif où diverses techniques aident à la lecture de ce personnage, à la découverte de son être intime. Une scène est mise en place permettant que les techniques entrent en force et la peuplent selon que les acteurs joueront ou non ce jeu, les laisseront faire ou non. 
Les techniques sont censées mettre fin à l'amateurisme et, ainsi, côté employeur, renforcer la certitude du diagnostic, réduire la dimension de pari fait sur la personne embauchée, accroître sa prévisibilité. La technicisation de l'embauche est portée par la professionnalisation de la fonction ressources humaines et, à l'intérieur de celle-ci, du recrutement. Celle-ci s'apparente à un désencastrement de cette fonction : de la même manière que le marché a, dans l'histoire moderne, fait l'objet de tentatives pour l'autonomiser à l'égard des règles sociales et politiques (Polanyi, 1944), cette professionnalisation consiste à justifier que la fonction relève de lois propres, avec des spécialistes, connaissant ces lois, capables d'agir au sein de départements respectant le périmètre de la sphère dessinée par ces lois. La constitution des premiers services centralisés du personnel (à partir des années 1920 en France, deux décennies auparavant aux Etats-Unis) (Cohen, 1993) a retiré l'embauche et le licenciement des mains des contremaittres pour en donner le contrôle aux directions d'entreprises qui ont été amenées à en faire une affaire de spécialistes. Avec l'affirmation des services du personnel dans la première moitié du $\mathrm{XX}^{\mathrm{e}}$ siècle puis « des dirigeants professionnels des ressources humaines » dans la moitié suivante (Fombronne, 2001), l'embauche, devenue "recrutement», est prise dans le "projet de professionnalisation" (Larson, 1977) des spécialistes des ressources humaines (les « RH ») et, parmi eux, des spécialistes du recrutement et d'experts divers (graphologues...). Ce mouvement de professionnalisation intègre à forte dose les recommandations d'utilisation de techniques, puisant dans des savoirs aux bases scientifiques plus ou moins reconnues (psychologie comportementale, cognition, graphologie...) et distinguant les experts de la fonction des profanes que sont les autres responsables au sein de l'entreprise. Dans leurs mains, de banals écrits comme la lettre et le CV sont devenus de puissants outils de révélation de la personnalité des candidats, de leurs aptitudes à occuper les postes, de la manière dont ils occuperont ceux-ci. Ils s'imposent comme des nécessités pour aider les recruteurs à venir à bout de leur tâche.

Les techniques traduisent alors la connaissance de ces lois de l'individu au travail (déterminants de la motivation dans les diverses personnalités...) et la possibilité de les repérer avant l'embauche. Il s'agit de donner à la sélection des fondements objectifs en lieu et place de l'appréciation subjective à laquelle se livrerait sans cela le patron profane, de produire de l'objectivité dans une rencontre supposée soumise aux aléas de l'inter-subjectivité. L'enjeu est de dépasser la rencontre de deux subjectivités en intercalant des médiations, faites d'humains et de non-humains : un examen de la lettre et du CV (sur le contenu, le style, les codes graphiques et d'agencement des rubriques du CV...) et de ce qu'ils révèlent, une mobilisation éventuelle de prestations de service de spécialistes, des tests de personnalité, etc. Le ou les en- 
tretiens font partie de la panoplie mais sous contrôle de savoirs qui dictent à l'expert ce qu'il doit y rechercher. A la limite, le consultant d'un cabinet de recrutement reconnaitra qu'il y a fait intervenir le feeling mais celui-ci finit de cautionner un processus suffisamment armé de techniques par ailleurs pour certifier que la sélection est faite en connaissance de cause, en ayant percé du mieux possible la personnalité du candidat.

\section{...et la liberté (relative) des acteurs}

La pression normative est certaine, en premier lieu, pour les candidats qui ne peuvent guère prendre le risque de réduire leurs chances. Les candidatures baroques ne sont autorisées qu'à titre très exceptionnel, lorsqu'elles sont le comble de la maitrise de cette obligation sociale comme cette candidate suédoise qui libelle ainsi son annonce : «Je veux un travail bien payé. Je n'ai pas d'imagination, je suis antisociale, pas créative et sans talent. » (Le Figaro, 23/4/2003). A en croire le journal, les propositions d'emploi auraient afflué. Cet acte ne tourne les impératifs sociaux en dérision qu'en apparence car il délivre précisément le message d'une personne audacieuse et imaginative. Ce n'est qu'un écart contrôlé à la norme.

De leur côté, les personnes qui embauchent n'ont pas beaucoup plus de liberté, notamment dans les grandes organisations où un responsable peut difficilement risquer un recrutement qui ne respecterait pas le processus attendu. Le secteur de la grande distribution représente, de ce point de vue, un espace de plus grande liberté. Les hypermarchés et les supermarchés ne se sont évidemment pas tenus complètement à l'écart de la tendance générale à la professionnalisation des ressources humaines mais les formes particulières de la gouvernance des unités économiques y exercent des effets particuliers, et plus encore pour les plus petits de ces magasins ${ }^{1}$. Même dans les enseignes d'intégrés ${ }^{2}$, les supermarchés ont toujours tendance à être conçus comme des entités économiques relativement autonomes : le directeur se décrit souvent comme un " commerçant», un "épicier », qui gère son affaire en respectant les « politiques » de l'enseigne mais sans grande immixtion de sa hiérarchie dès lors que «les chiffres » sont là. C'est lui qui embauche les caissiers et employés commerciaux, et non un service RH. Les chefs de rayon et les directeurs de magasins sont recrutés par la direction régionale

1. Rappelons que les supermarchés sont des grandes surfaces à dominante alimentaire d'une taille comprise entre $400 \mathrm{~m}^{2}$ et $2500 \mathrm{~m}^{2}$, tandis que les hypermarchés sont des grandes surfaces de plus de $2500 \mathrm{~m}^{2}$

2. Les groupes de la distribution à prédominance alimentaire sont, soit des regroupements d'indépendants (chaque magasin a un propriétaire), soit des « intégrés » (le directeur du magasin est un salarié de l'enseigne). 
des ressources humaines mais celle-ci, dans l'enseigne étudiée, est composée de responsables ressources humaines qui revendiquent également leur identité de "commerçants ", de gens ayant fait l'essentiel de leur carrière en magasins ${ }^{1}$.

Les directeurs de supermarchés n'ont pas la contrainte de devoir justifier à un tiers les critères et les modalités de leur décision d'embauche. Ils en tirent une liberté à l'égard des normes de professionnalisation de cette embauche, intéressante par ce qu'elle révèle de la manière dont la logique des usages peut s'imposer face au dispositif et mettre en tension la part concédée au technique.

Leurs représentations et leurs comportements donnent un relief également intéressant aux présupposés des candidats tels qu'ils se laissent observer dans le corpus de courriers analysés. Les postulants se montrent globalement conscients de la norme à atteindre mais l'adhésion n'est pas uniforme et, par ailleurs, la maitrise de la technique est très variable. Du jeu existe donc aussi de leur côté.

\section{Le dispositif dans les mains des utilisateurs}

Dans les hypermarchés et les supermarchés du groupe étudié, les candidatures collectées par Internet occupent encore une place réduite. Etre alimenté par de telles offres chaque matin ne fait pas encore partie du quotidien d'un directeur de supermarché qui opère dans un univers relativement inchangé en la matière : candidatures reçues au courrier ou déposées à l'accueil, annonces collées sur la vitrine ou passées dans la presse locale, etc. La DRH de l'enseigne réfléchit aux possibilités de développer l'utilisation d'Internet mais sans paraitre y voir un futur immédiat. Les supports de la rencontre entre postulants et recruteurs demeurent donc les classiques lettres de motivation et $\mathrm{CV}$.

\section{Deux imaginaires de l'écrit}

\section{Celui des candidats}

La lecture des courriers fait apparaitre des candidats globalement soucieux de bien faire et qui, dès lors, confortent la dimension technique du dispositif : raffinement de la mise en page du CV, photographie, lettres travail-

1. Les hypermarchés, quant à eux, sont soumis à une plus grande centralisation : les magasins sont tenus par un plus grand nombre de procédures uniformisant leurs pratiques. Dans les hypermarchés du groupe étudié, le siège concentre des spécialistes des ressources humaines qui sélectionnent les candidatures. Encore ont-ils, eux aussi, le profil d'anciens opérationnels des magasins bien que davantage marqués par l'identité spécifique des hommes du siège. 
lées pour présenter, parfois, une argumentation extrêmement construite, etc. Cela n'exclut pas que certains ne voient ces documents que sous leur aspect le plus fonctionnel, c'est-à-dire ne ressentent pas l'obligation de coopérer à la production d'un jugement sur leur personne, de contribuer à l'outillage des décisionnaires :

" Je sollicite de votre bienveillance un emploi dans votre société. Vous trouverez joint à la présente mon curriculum vitae.

En l'attente d'une réponse favorable de votre part, veuillez agréer, Madame, Monsieur, (...).»

Mais la plupart des rédacteurs ont bien compris l'utilisation qui est faite de leurs écrits et s'efforcent d'entrer dans le jeu. Ils collaborent à la dimension technique en fournissant de quoi être analysés. Car telle est la contrainte pour voir son dossier sélectionné parmi la masse de ceux que reçoit l'entreprise. Une autre chose est la performance de l'exercice, soit en raison de caractéristiques du candidat (parcours chaotiques, par exemple), soit d'une maittrise insuffisante (maniement difficile de l'écrit...).

Les jeunes diplômés sont ceux qui contribuent le plus à renforcer le dispositif socio-technique par des envois très élaborés sur le plan de la conception (notamment graphique) des CV et de la démonstration de la motivation dans la lettre. Maitrise des technologies informatiques, connaissances en matière de communication, culture marketing ambiante, conseils reçus à l'école : tout cela y concourt probablement. Ils butent cependant sur le manque d'expériences professionnelles, qui limite la performance de leurs écrits. La rhétorique de la motivation à laquelle ils sont réduits intensifie leur adhésion à la logique de perfectionnement technique de la candidature.

Dans ce cadre, les courriers consistent en majorité en une entreprise de communication sur soi, de promotion de sa personne dans ses capacités professionnelles, sa personnalité, voire ses valeurs éthiques personnelles ( Femme de conviction et de contact, j'aime m'investir dans ce que j'entreprends »; « fédérateur dans l'âme »). Cette entreprise se destine à un lecteur qu'un très grand nombre (et plus encore lorsqu'on tend vers les postes de cadres ou les jeunes diplômés) se représente sous les traits d'un responsable des ressources humaines (explicitement dans la désignation du destinataire de l'envoi ou implicitement dans la façon de rédiger). C'est bien là une des sources principales de difficulté qu'affronte le rédacteur que cette obligation de construire un destinataire absent, de se figurer le récepteur du courrier, ce qu'il attend d'un envoi de ce type, ce qu'il lui plaira d'y lire, ce qui le convaincra. Le stéréotypage, « opération qui consiste à penser le réel à travers une représentation culturelle préexistante, un schème collectif figé » (Amossy, 1999:135), joue pleinement dans cette identification de l'interlocuteur au 
professionnel des ressources humaines. On sait, ou on pense savoir, qu'un tel personnage a certaines attentes : recevoir une sollicitation montrant l'intérêt que l'on porte à l'entreprise, la connaissance qu'on en a (les candidats parlent souvent de leur enthousiasme pour ce "leader de la distribution » qu'est l'entreprise, citent son chiffre d'affaires, etc.), parler de ses qualités, de son dynamisme, clore la lettre par la demande d'un entretien.

«X est leader de la grande distribution en France. Par une gestion visant la rentabilité et les performances commerciales, par la mobilisation de moyens humains et par l'écoute constante de sa clientèle, votre société parvient à la satisfaire et à la fidéliser. C'est pourquoi j’aimerais travailler au sein de votre société en tant que manager métier. Grâce à ce poste, je pourrais gérer l'ensemble d'un rayon d'un de vos magasins en étant sur le terrain et manager une équipe de personnes pour atteindre les objectifs économiques qui me seront fixés ».

\section{Celui des directeurs}

L'examen isolé des courriers montre des candidats en train de se débattre avec le sentiment d'une obligation de communiquer sur eux-mêmes, bref de se débattre avec le dispositif socio-technique tels qu'ils pensent le connaitre et le maitriser. Mises en regard des pratiques de recrutement des directeurs de supermarchés, ces attitudes des postulants prennent encore plus de relief, car les directeurs mettent à l'épreuve le dispositif socio-technique. Ils ne se le laissent pas imposer sans réagir n'en sélectionnent que ce qu'ils souhaitent conserver et, pour résumer, tendent à le dépouiller assez sensiblement de ses prétentions techniques. Précisément, pour eux, il est hors de question de le laisser fabriquer cette communication sur soi des candidats qui n'est que du bruit perturbant l'identification de leur personne authentique.

Les directeurs de supermarchés réalisent l'embauche des caissières et employés de rayons. Ils assument cette tâche parmi une multitude d'autres. Ils entendent faire comprendre à l'enquêteur qu'ils sont des généralistes de la direction des magasins, non des professionnels du recrutement. Ils tirent argument de leur fonction pour faire admettre qu'on ne saurait les appréhender dans leur pratique de l'embauche avec les critères, les exigences, que l'on opposerait à un spécialiste des ressources humaines. Ils concèdent sans difficulté ne pas maitriser les techniques RH et ne ressentent pas le besoin de s'en défendre.

"Q. Donc, en fait, la part de feeling est quelque chose d'assez, important?

R. Oui.

Q. Et difficile à contourner. Il n'y a pas des outils? 
R. Si, y a des outils, mais alors, après, c'est au niveau RRH, DRH. Mais, je veux dire, j'ai pas l'temps de passer... Euh, un RRH, il vous passera deux heures, deux heures et demi avec un... Moi, il faut qu'en vingt minutes, maximum, ce soit fait. ».

Le décor est posé : le temps du directeur étant compté, il ne s'embarrassera pas de tout ce qui cautionnerait la sélection. Le dispositif n’a pas besoin de s'étoffer de techniques prétendant objectiver la décision. Les candidatures spontanées conservées ne font pas l'objet d'un archivage organisé mais, au contraire, guidé par l'optimisation du temps (on les accumule puis, régulièrement, on fait du ménage dans les courriers les plus anciens, sans distinction); lors d'une embauche, le tri de ces candidatures repose sur un examen assez rapide de la lettre et du CV, essentiellement avec la préoccupation d'éliminer les postulants jugés les moins fiables (abandons d'emploi trop fréquents...) et d'obtenir les informations déterminantes (disponibilités horaires ....). L'entretien, quant à lui, ne s'étend guère, la plupart du temps, au-delà des vingt minutes.

L'écrit fait, chez eux, l'objet d'une véritable défiance. D'une manière générale, et plus encore pour ceux qui revendiquent un parcours d'autodidacte, ils voient dans l'écrit le lieu possible de l'enjolivement, pour ne pas dire de la duplicité. A la limite, tout rédacteur est un faussaire en puissance.

Ils paraissent toujours en déficit de preuves de sincérité, toujours sur le qui-vive, anticipant le risque d'une fausse motivation, d'un écrit qui serait contredit par la réalité de la personne. Avec un tel état d'esprit, la « professionnalisation » des candidatures construite par les manuels se retourne, à leurs yeux, en fabrique de faussaires professionnels :

«J'ai horreur des lettres stéréotypées. (...) J'ai horreur des lettres du genre: "Votre groupe étant connu pour, euh..., sa vitalité extraordinaire"...».

" Je vous ai dit... L'écriture, l'envie aussi qui est venue du contenu lui-même. Si c'est des trucs génériques classiques "Je suis très motivé, gna, gna, gna...", là aussi, il est pas plus intéressé par moi que par les autres et puis, il s'est pas emmerdé non plus. Il m'a fait un truc passe-partout; ce qu'on va lire dans tous les bouquins à quatre sous, Rédigez votre $C V$ ou autre. Je vais pas le prendre en priorité ».

Les directeurs se révèlent ainsi particulièrement méfiants à l'égard de ce qui est produit par la professionnalisation des candidats. Leur tendance à conformer leurs écrits à des normes toujours plus stéréotypées finit par être contreproductive et à rendre tendanciellement semblables les différents dossiers. Cette uniformisation les gêne. Elle est identifiée à une montée de l'arti- 
fice, du factice, par ceux qui recherchent en priorité les vertus inverses : la vérité, l'authenticité.

C'est l'écrit qui constitue ici une pierre d'achoppement : comment, par ce seul moyen qui ne compense qu'imparfaitement la rencontre directe avec l'interlocuteur, informer efficacement celui-ci sur la personne que l'on est, construire la conviction auprès de lui, créer la confiance ? Comment se rendre suffisamment concret ? Comment s'ajuster à ses attentes, tenir à la fois ce que l'on est et s'adapter à ses souhaits ? Les courriers montrent des candidats qui n'ont d'autre ressource, quand ils en sont capables, que de travailler l'écriture pour lui faire porter toute la charge, tout l'effort de conviction. On voit certains tentés d'en rajouter sans cesse pour manifester leur sincérité quitte à allonger considérablement la lettre ou à pratiquer la redondance :

«Ma volonté et ma détermination de travailler dans un des centres de $X$ est très importante, c'est pour cela que mon engagement et mon dévouement n'auront d'égal qu'à travers (sic) la disponibilité et l'écoute qui seront les miennes pour apprendre et acquérir une réelle technique et expérience commerciale qui me sera essentielle pour la suite. »

On ignore quel est le destin de telles tentatives. On comprend plutôt, en écoutant et observant les directeurs de supermarchés, qu'il n'existe pas d'algorithme général - lequel permettrait d'approcher l'ambition des manuels de construction d'une prévisibilité -, mais bien au contraire l'effet imprévisible, incontrôlable, de la lecture qu'un sujet peut en faire. En fonction de son identité, elle-même profondément marquée par sa trajectoire, les milieux sociaux d'appartenance, ce sujet prend en main le courrier avec une certaine liberté d'interprétation, un certain engagement de valeurs, une disposition à s'agacer ou à se montrer sensible à des points qui ne feront pas réagir un autre de la même manière. Les directeurs de supermarchés marquent, de ce point de vue, leur différence avec les spécialistes RH. Ils ne cherchent pas à se construire contre ceux-ci et leurs outils, ils n'en dénient pas l'utilité ou l'efficacité, mais tiennent ferme sur leur propre approche à eux. Approche plutôt que méthode : ils recourent au feeling, ils le revendiquent, mais ils ne cèdent aucunement à la sollicitation du chercheur d'entrer dans l'analyse de ce qui constitue ce feeling.

\section{Le feeling ou le jugement par-delà l'écrit}

Comme le souligne E. Marchal (2005:130), le recruteur professionnel est tenté de se doter d'outils d'évaluation objectivant sa décision, tant parce qu'il « est plus sûr de lui si ses impressions ne sont pas uniquement personnelles, acquises lors de face-à-face » que pour la généralité et ainsi la crédibilité de son évaluation auprès de son client. Les consultants en recrutement ne se 
privent pas du feeling, des impressions laissées par le candidat lors de la rencontre de visu (Eymard-Duvernay et Marchal, 1996) mais ils ne le présentent jamais comme l'argument décisif, confié à un dispositif mobilisant des outils d'apparence plus scientifique.

Travaillant directement pour eux-mêmes, se voulant généralistes de la direction de magasins et non professionnels du recrutement, les directeurs de supermarchés s'émancipent de ces contraintes et se sentent libres d'afficher le rôle prédominant qu'ils attribuent au feeling. Ce n'est pas qu'ils ignorent le dispositif socio-technique élaboré. C'est plutôt l'affichage résolu de leur conviction des limites de celui-ci et, réciproquement, de la confiance qu'ils estiment pouvoir raisonnablement accorder à leur propre dispositif apparemment rudimentaire, mais finalement bien ajusté aux objectifs.

Ce jeune directeur d'un magasin parisien, par exemple, connaît très bien le dispositif le plus technique... pour en avoir lui-même été victime :

«Et puis j'ai tellement été déçu des recruteurs pendant la période où je cherchais du boulot. Elles sont nulles; elles sont toutes plus nulles les unes que les autres. J'étais confronté à des gonzesses de vingt-cinq ans, qui avaient fait deux ans de sociologie, qui avaient jamais bossé en entreprise, qui savaient pas ce qu'était qu'un commercial ou un rendez-vous clientèle ou un entretien de portefeuille de clients et qui me posaient des questions de merde et qui me jugeaient, mais je sais pas sur quoi. Je ne recrute absolument pas de la même manière que ces personnes-là, je fais table rase de tout ce qui est... fausse idée, interprétations... (...) j'essaie de pas me focaliser sur des études, des trucs, j'essaie vraiment de voir les qualités humaines de la personne. Ça ça m’a beaucoup déçu quand j'étais en entretien parce que je...

Q. (...) Pour ces qualités bumaines, y a pas de batteries de tests, de procédures scientifiques pour les mesurer?

R. Non, c'est un peu au feeling. Je leur pose certaines questions : "Qu'est-ce que vous feriez dans telle situation ? Comment... vous voyez qu'un employé, un de vos collègues, vole ; vous faites quoi ?" ».

Cet homme se trouve alors assez proche des positions de ses homologues, y compris les autodidactes qui affichent volontiers un parti pris anti-écrit, l'excès en moins : il ne rejette pas l'écrit en général mais n'exige des postulants que celui qui sera directement utile dans le travail. Ainsi livre-t-il les candidats, le jour de l'entretien, à une petite dictée, suivie de quelques opérations de calcul, pour vérifier ce à quoi il peut s'attendre.

Le problème de fond de la communication, le défi principal que doit surmonter le dispositif de rencontre, est de produire de la confiance, alors que les vecteurs de celle-ci ne sont pas totalement prévisibles, profondément dé- 
pendants qu'ils sont des grilles de lecture que le destinataire active pour détecter un candidat digne de confiance. On ne produit pas de la confiance de la même façon selon qu'on s'adresse à un professionnel RH ou à un directeur opérationnel. Les signes de confiance sont loin d'être univoques et chacun a ses propres règles pour les détecter. Là où les directeurs mettent en doute la capacité du dispositif socio-technique des spécialistes, c'est dans ce qu'il produit comme information permettant de fonder la confiance : plus ce dispositif s'élabore, plus il renforce la dimension de communication sur soi des candidats, et moins, par conséquent, il s'avère productif. Les directeurs estiment qu'il brouille le message, éloigne de la compréhension de la " vraie » personne. Percer la réalité de celle-ci, de ce qu'elle sera dans son travail, est le cœur du processus de décision, mais avec cette spécificité que les directeurs le font en construisant un personnage qu'ils rapprochent d'individus dont ils ont fait l'expérience, positive ou négative, dans leur parcours professionnel.

C'est là que le dispositif socio-technique des spécialistes atteint pour eux ses limites : il amène les candidats à se composer un portrait de travailleur idéal parfois éloigné de la réalité. Les directeurs cherchent à mettre en correspondance une personne avec un poste, des caractéristiques concrètes duquel ils ont une connaissance assez intime : être employé dans un rayon " épicerie sèche » induit une série de gestes, d'actes, de décisions, d'attitudes matériellement très distinctes de celles de l'employé du rayon parfumerie ou de la poissonnerie. Dans un cas, la journée sera composée de moments où il faudra manipuler des boîtes de conserves : c'est lourd mais le linéaire est plus rapidement composé que dans le second rayon, qui demande d'aligner patiemment de petits objets, et c'est encore bien différent de l'humidité et du froid, des mains dans la glace, du dernier d'entre eux. L'appréciation pour l'embauche consiste à projeter l'individu dans le poste, avec les implications concrètes de celui-ci. Elle suppose la collecte d'indices qu'il faut faire parler et qui sont masqués par la communication sur soi :

«Le gars qui arrive avec un costume Armani ou quoi que ce soit, ah, putain, le mec il va pas travailler en rayon, c'est pas possible. Une fois, j’ai eu ça, le mec, il avait un costard avec le logo Versace. Je le regarde et je lui dis "Vous cherchez vraiment du travail ?", et il me fait "Oui", et je lui dis "Le costume, vous l'avez ou vous l'avez pris à votre père, jeune homme ?". Il me fait "Non, monsieur, il est à moi, pourquoi ?". "Et, dans la vie, il va falloir travailler et vous allez travailler en Versace". »

Les inférences faites par les directeurs en les comparant aux postes de travail et à des personnes en chair et en os qui les ont tenus les amènent à s'interroger sur les dispositions et les capacités effectives que manifestera la personne à tenir durablement sur ces postes. Les qualités d'une telle per- 
sonne se paient raisonnablement de défauts sur d'autres points, un individu concret étant nécessairement constitué ainsi.

C'est ce que le feeling, le jugement intuitif fondé sur une expérience des personnes réelles telles qu'on les rencontre, a pour charge de déceler. C'est l'information qu'il doit produire. Ce qui revient à dire que les directeurs, méfiants à l'égard de ce que peut fabriquer la professionnalisation des candidats, ne prétendent pas à une absence de dispositif socio-technique mais revendiquent, de fait, un dispositif amateur pour son efficacité comparée vis-à-vis du dispositif professionnel. Qu'on les laisse gérer leurs embauches en amateurs, puisqu'ils ne font pas profession de recruter et ont bien d'autres préoccupations à faire aboutir dans une journée et que, ayant, par la suite, à faire avec les individus recrutés, ils préfèrent les avoir choisis en connaissance de cause, cette connaissance que produit mieux que tout le feeling.

\section{Amateur mais dispositif socio-technique quand même}

Toute rudimentaire qu'elle apparaisse, l'embauche réalisée par les directeurs de supermarché se réalise donc au moyen d'un dispositif socio-technique, un agencement confectionnant les bases de la confiance permettant de prendre la décision d'embauche, de ne pas rester paralysé par le mystère de la personne qui a postulé.

\section{Un dispositif qui fait confiance à la sociologie des sens}

Caractéristique essentielle de ce dispositif : il relativise le rôle de l'écrit, il le limite pour éviter les pertes d'informations qu'il fait subir, et, au contraire, il maintient sa confiance à la part d'intersubjectivité en accordant le rôle primordial à la rencontre de visu. Autrement dit, ce dont on s'arme, à l'intérieur du dispositif amateur, c'est des ressources de la sociologie des sens telle que la décrit G. Simmel :

«Dans une certaine mesure, très variable il est vrai, nous savons dès le premier coup d'œil à qui nous avons affaire. Si ce fait et son importance fondamentale nous échappent, cela vient de ce que nous détournons tout de suite notre attention de cette base évidente pour la fixer sur la reconnaissance de certains traits particuliers, de certains détails singuliers qui servent à déterminer notre attitude pratique vis-à-vis de chacun d'eux. Mais si, au contraire, nous cherchons à pénétrer plus avant dans la conscience que nous avons de cette base évidente, nous sommes étonnés de tout ce que nous savons d'un homme dès le premier coup d'œil que nous jetons sur lui. Et ceci n'est rien qui puisse s'exprimer par des 
concepts, rien qui puisse s'exprimer par des paroles. » (Simmel, 1981:229).

L'écrit ne peut rivaliser avec cette impression des sens, porteur qu'il est de deux défauts majeurs. D'une part, il est vecteur d'abstraction dans un moment où l'on doit, à l'inverse, se faire une représentation concrète de l'interlocuteur comme être réel perçu par les sens. Les directeurs ne sont pas intéressés par un être abstrait mais par un être que l'on projette en situation. D'autre part, l'écrit favorise une certaine uniformisation de l'usage du langage : il réduit l'infinie variété de formulations qu'offre l'oral et, ce faisant, obscurcit plus qu'il ne dévoile une individualité et ce a fortiori dans le cas des écrits fonctionnels que sont la lettre et le $\mathrm{CV}$ qui relèvent comme disait $\mathrm{R}$. Barthes de la «scription » plus que de l'écriture. L'écrit prolonge la situation de communication où les deux protagonistes ne sont pas présents physiquement l'un à l'autre et qui exclut donc tout recours à d'autres codes sémiologiques que le langage lui-même. Loin de doter d'outils celui qui doit se former un jugement, il le prive des codes gestuels (renforçant généralement l'usage du langage) et des indications identitaires et sociales (âge, niveau social, origine ethnique), psychologiques (inquiétude, nervosité, sérénité, agressivité...) ou encore de la manière dont l'interlocuteur se conforme aux règles de civilité, aux rôles attendus dans une situation d'embauche... En ce sens, l'écrit décontextualise et généralise quand c'est l'éclairage d'une singularité qui est recherché. Charger le dispositif en technique, c'est le vider de l'information sociale dont il est porteur alors que l'intersubjectif sera au cœur du travail (relations avec les collègues, la hiérarchie, les clients).

Les ressources sémiologiques issues de la combinaison des systèmes de signes (gestuelle, habillement, expression verbale, respect des codes sociaux...) sont au cœur de ce dispositif qui voit dans l'écrit un équipement peu productif et qui attend beaucoup plus du face-à-face. Ce dispositif assume la contrainte de ne pas automatiser la prise de décision, de ne pas la confier à une mécanique, mais de faire effectuer le principal du travail à la personne qui embauche, obligée de se prêter activement au décryptage de ces signes, de faire travailler ses sens.

Au besoin, les directeurs renverseront le dispositif socio-technique professionnel et valoriseront les ratés dans l'écrit, qui peuvent devenir une chance pour certains candidats. La maladresse peut être un signe de sincérité parce qu'elle trahit une spontanéité, qu'elle est l'anti-duplicité. C'est du moins ce qui s'est passé avec Adriana selon ce directeur qui se félicite de l'avoir embauchée. Tout dans son courrier exposait Adriana à voir rejeter sa candidature par un dispositif d'embauche exigeant la professionnalisation des candidats : elle s'était contentée d'une lettre sur une feuille d'écolier à grands carreaux. Elle y avait ajouté, en entretien, de venir chaussée négli- 
gemment. Mais postulant dans un supermarché, sa candidature a été lue par un directeur qui n'en a pas fait cas, d'autant qu'il est de ceux qui revendiquent leur histoire d'autodidacte :

«On m'a pas formé à lire les CV bien faits avec les cases grises, machin... Je regarde pas du tout la forme du CV, si c'est bien saucissonné... Elle [Adriana] a joué franc jeu; en voyant le papier [à gros carreaux], je me suis dit "elle va pas me raconter une connerie". C'était vrai ; elle était en face de moi, elle m'en a pas raconté une. C'est franco. Une pile, par contre, une vraie pile. $\mathrm{Y}$ en a des gens qui sont francs. $\mathrm{Y}$ a trop de faux culs, d'hypocrites, j'aime pas. C'est le genre d'entretiens où il faudrait les prendre en photo, c'est le jour où ils sont toujours les mieux habillés. A $90 \%$, c'est ça. Y a un mec, il arrive avec un costard ; y en a c'est le tailleur, super maquillées ; c'est toujours ça. Adriana, elle s'est ramenée avec ses tongs, son pantalon, comme elle était et puis voilà. C'est franco, c'est pas je me déguise, je vais essayer d'embobiner quelqu'un. Tout ça, ça marche pas. ».

La performance d'une candidature ne suit pas un destin pré-déterminé par son respect d'un dispositif, notamment le dispositif professionnel. Elle peut même échouer par le simple fait que, conçue en se référant à un tel modèle, elle entre dans un circuit d'appréciation recourant au dispositif amateur. L'invocation de l'univers domestique, des références de proximité comme dans le cas suivant, «Tout cela je l'ai déjà démontré dans votre structure. Vous pourrez d'ailleurs vous renseigner auprès de Marie-Paule M., Marie-Claire P. ou Jocelyne M. », peut alors se montrer tout aussi efficace que l'affirmation de son «dynamisme».

\section{Là où les deux dispositifs peuvent se rejoindre}

Pour autant, dispositifs amateur et professionnel ne sont pas complètement exclusifs l'un de l'autre. Dans le cas où le second accepte d'intégrer l'entretien dans sa panoplie d'outils, il reconnait bien, à travers lui, l'équipement que fournit le dispositif amateur à la formation du jugement et à la prise de décision. L'entretien, lorsqu'il a lieu, témoigne du fait que c'est en faisant parler la personne et en la voyant se manifester que le mystère peut se dissiper un peu plus.

L'entretien est une conversation par laquelle, selon une logique assez proche de celle de l'entretien sociologique, le candidat est insensiblement amené, en formulant des dires et en donnant des informations, à livrer des indices sur lui-même. Sans que le profane le voie, celui qui conduit l'entretien est comme en train, par ses questions, de remplir, sur diverses rubriques, des colonnes actif et passif. 
Nous allons regarder travailler un responsable de ressources humaines lors d'un entretien de pré-recrutement qu'il fait passer ici à deux candidats. On l'a dit, dans cette enseigne, ces RRH sont généralement d'anciens opérationnels des magasins qui ne se sont que partiellement acculturés à la discipline RH. Dans les deux cas, il s'agit de décider si le postulant pourra, un jour ou l'autre, occuper une fonction managériale. Dans les commentaires qu'il livre après chacun des entretiens, le RRH montre comment, d'une certaine disponibilité à entendre des arguments favorables et défavorables à cette hypothèse (simplement pré-orientée par la lecture de la fiche remplie par les postulants), il accumule des indices dans les deux directions et voit dans quel sens l'accumulation se fait.

Premier candidat : un homme d'environ vingt-cinq ans qui a travaillé chez Metro mais n'a pas pu y faire carrière.

Le RRH lui pose des questions comme «Est-ce que vous avez pu savoir pourquoi, pour eux, vous n'étiez pas évolutif ? ", "Vous, concrètement, aujourd'hui, quel est votre projet professionnel ? », «Vous vous êtes fixé un délai pour accéder au poste de directeur de magasin ?».

Il commente ainsi :

«Metro, c'est l'échec, c'est un échec.

\section{Q. Mais comment vous expliquez, qu'on lui ait donné sa chance?}

R. C'est pour ça que je lui ai posé la question. Parce que vous voyez comment ça se passe ; il a fait Arcat Formation ; c'est tout simplement le partenaire référencé par Metro pour faire les promotions ; ils lui ont fait un contrat en alternance qui lui permettait de passer son BTS Action co', ils lui ont donné sa chance par contre ils ont dit "Il en veut, on va essayer avec lui". A priori, le contrat de qualification, ça c'est bien passé, mais, après, il faut qu'il développe une réelle motivation pour qu'on puisse lui donner des responsabilités managériales. Et là, ils lui en ont pas donné. Il l'a dit ou laissé dire. Dans le management, il a pas de puissance ; il a pas de puissance managériale. Il a une connaissance de la grande distribution, ça c'est vrai ; je l'ai noté d'ailleurs, il est très tourné vers la tâche, il a l'indépendance déjà. Il est pas tourné vers le collectif, c'est un indépendant, il est tourné vers lui-même. (...) Vous l'avez compris, pour moi, c'est non. Je vais essayer de développer. Il a eu sa chance ; on lui a donné réellement sa chance mais il l'a pas saisie. On le voit bien il cherche sa voie. Quand je lui ai posé la question, s'il avait fait d'autres démarches... "J'ai écrit aux banques"... Pour moi, il a pas transpiré la motivation pour la grande distribution. La banque, il recherche autre chose dans la banque. Quelqu'un qui est fait pour la grande distribution, il va pas à la banque; nous sommes opposés, nous sommes complètement opposés, 
d'accord. La banque effectivement y a pas de travail d'équipe, vous êtes un indépendant...on va peut-être lui proposer un poste de conseiller financier, encore une fois, ça correspond à sa personnalité ; vous allez vendre un produit... il l'a dit sur le management... sur le management, c'est là où ça allait pas ... j’ai repris, j'ai rebondi... je l'ai posée une première fois, puis une deuxième fois...

\section{Q. Il a un discours technique...}

R. Il veut pas se faire piéger, il est pas confiant ; il le sait que c'est un échec. Par contre, il est honnête... il est honnête, il est sincère... Par contre, il est pas fait pour le management, à la limite pour la gestion, ce serait un bon gestionnaire de vente mais le poste existe plus aujourd'hui dans la grande distribution. Applicateur, "tu fais ça... tu fais ça", tu recomptes derrière...

\section{Q. C'est quelque chose qui peut pas s'acquérir?}

R. Moi, au terme de six mois, non. Par contre, effectivement, il peut évoluer. Pour le voir évoluer, à la limite, demain matin je lui propose un poste d'employé... Je lui fais passer un CQP, un certificat de qualification professionnelle pour l'aguerrir sur des connaissances commerciales de gestion mais pas manager de rayon... Dans six mois, il est pas manager de rayon... ça par contre, c'est clair, il managera pas une équipe. ».

Second candidat. Vingt-trois ans, il répond timidement, souvent par monosyllabes. Son père tient une supérette en province.

«Vous l'avez peut-être compris, je posais beaucoup de questions techniques c'était pour voir véritablement de quelle manière il aidait son père. Vous avez beaucoup de fils de partenaires qui entre guillemets ont été élevés dans le coton, ils connaissent pas les réalités de la grande distribution, ils connaissent pas les soucis du commerce, parce que le commerce c'est quand même quelque chose d'ingrat... on est au service du client; il faut être aimable, serviable, c'est des heures surtout dans les petits commerces de proximité. Il faut être là le matin. Donc, c'est de voir exactement ce qu'il avait vu... On voit bien son père il lui a montré tous les côtés du métier, aussi bien les avantages que les inconvénients du métier... il a du mal à mettre en place, en forme ses idées quand même... il a un peu du mal.

\section{Q. C'est pas théorisé.}

R. Ah non, pas du tout... il a peu de recul. C'est quelqu'un qui agit et qui réfléchit moins entre guillemets. Il réfléchit, c'est pas ce que je veux dire. C'est plus un applicateur qu'un... Mais dans le temps il peut évoluer... 
Applicateur dans le sens où il est jeune, attention, c'est pas ce que je veux dire. Il a un potentiel d'évolution, mais ça c'est l'avenir qui le dira...

(...) Là c'est un jeune que je peux mettre sur Paris ; c'est déjà bien ce qu’il fait, il est prêt à partir à déménager, il a un bon côté quand même, il a vingt-trois ans...

$(\ldots)$

Q. (...) Comment apprécier le potentiel à partir du peu d'informations qu'il vous a données?

R. Oui, c'est en mettant un pied dans l'entreprise qu'on va le voir. Le potentiel qu'il peut avoir... manager de rayon, il peut y accéder, il faudra voir avec le deuxième entretien. Il peut y accéder...

Q. Déjà le faire évoluer vers un autre format ${ }^{1}$ que celui qu'il a connu...

R. Voilà, complètement... voir comment il réagit... mais vous avez des jeunes qui se révèlent par la suite. $Y$ a des jeunes au départ, il a vingt-trois ans, c'est sûr qu'il est moins à l'aise. Par contre, il est ambitieux... Y a des bonnes choses chez lui. Par contre, je sais pas du tout aujourd'hui je peux pas le projeter ; je peux pas me dire "Demain matin, il sera directeur de magasin". Je pense que s'il a le potentiel de passer directeur de magasin ce sera pas avant sept ans, ça c'est clair...

Q. Il doit acquérir de la maturité?

R. La maturité professionnelle. (...) Il est quand même déterminé pour vingt-trois ans. Je vous dis ça parce que je reçois beaucoup de jeunes diplômés et je fais la différence si vous voulez... (...) Par contre, c'est son ouverture... J'ai eu du mal...un deuxième entretien pour l'affiner. ».

Le jugement n'est pas définitif. Il cherche à saisir la personne en la voyant comme un kaléidoscope, qui, selon les jeux de miroir, se présente très variablement. On peut ne pas avoir de capacités managériales et être honnête. Cela veut alors dire qu'on n'est pas définitivement condamné mais qu'on peut se voir proposer un poste en dessous de celui pour lequel on postulait tandis que l'avenir dira comment on pourra évoluer. Inversement, on peut ne pas faire une démonstration clinquante, ne pas se montrer grand communicant. Si cela peut être mis au compte de la très grande jeunesse pour un candidat ayant pour lui d'avoir baigné dans la grande distribution par l'univers familial et ainsi vu le métier y compris sous ses aspects difficiles, alors l'indulgence s'empare du décideur.

1. Un format est un type de magasin : supérette, supermarché, hypermarché. 


\section{Conclusion}

Les développements précédents visaient à remettre la technologie Internet en perspective, aussi bien vis-à-vis des techniques dans un sens large (la technologie Internet intervient alors que des processus de technicisation de l'embauche sont en cours depuis plusieurs décennies) que vis-à-vis des marchés du travail non qualifiés alors que la numérisation du recrutement concerne, pour l'instant, de manière privilégiée les populations les plus qualifiées.

L'irruption d'Internet dans le champ du recrutement ne vient certainement pas faire table rase d'un passé prétendument non technologique. Tout d'abord, ce champ n'était pas dépourvu de techniques, en l'occurrence celles que les professionnels des ressources humaines se sont attachés à développer pour rendre plus efficace leur travail de prospection et de sélection des candidatures, des techniques qui se sont imposées aux postulants eux-mêmes. Tout cela ne s'opère pas, cependant, sans une appropriation de ces techniques par les postulants aussi bien que par les recruteurs. Pour ce qui est de ces derniers, tout spécialement, dans un secteur comme la grande distribution alimentaire, où l'espace conquis par les professionnels RH est en partie resté subordonné au pouvoir des opérationnels des magasins, ces techniques sont soumises par les directeurs de supermarchés à un important examen critique.

Dès lors, même si cela paraît une banalité de l'affirmer, il faut rappeler qu'Internet, pas plus que d'autres technologies, n'entraîne de déterminisme. Certes, la numérisation du recrutement, en intervenant, par exemple, sous la forme d'une alimentation des directeurs de supermarchés en candidatures placées par le biais d'un masque normalisé, imposerait à ces derniers les cadres de pensée RH auxquels ils sont encore largement en train de résister ${ }^{1}$. La numérisation ne sera donc probablement pas neutre dans les rapports de force autour de la définition des pratiques et critères légitimes de recrutement, et il est fort possible qu'elle fragilise la conception artisanale, ou amateur. Elle forcera sans doute davantage qu'actuellement les directeurs de supermarchés à se couler dans un moule auquel ils ont encore aujourd'hui largement les moyens d'échapper. Mais le problème qu'ils opposent actuellement au dispositif professionnel demeurera très probablement : Internet n'est rien d'autre qu'une forme de médiation écrite qui offre parfois des possibilités de dépassement des limites de l'écrit (les réseaux sociaux numérisés évoqués dans ce numéro en constituent peut-être un exemple) tout en

1. Cette remarque nous a été fort utilement adressée par Godefroy Beauvallet. 
conservant, malgré tout, ses limites. En outre, si la candidature par Internet se trouvait à induire une réduction de l'importance de la lettre de motivation au profit du CV, cela irait dans le sens des intuitions des directeurs.

La question, pour les acteurs, est de savoir quelle dose de technique ils doivent respecter pour que puisse être mesurée la valeur du candidat, pour qu'il puisse être jaugé. L'écrit peut être plus ou moins travaillé par la technique, par laquelle s'expriment des prescriptions, des normes, que les agents de la professionnalisation du recrutement s'efforcent d'imposer aux postulants et à ceux qui embauchent. Le dispositif peut ainsi se renforcer ou s'alléger en dimension technique, en liaison avec la liberté que les deux protagonistes principaux gardent à l'égard du dispositif, leur idée de la manière dont un surcroît de technique les aidera ou non à réaliser leurs intentions : pour le candidat, se faire bien juger et, pour le recruteur, bien juger. Le dispositif intervenant sur du matériau humain (la personne des postulants), les promesses de la technique de résoudre le problème qu'il représente (l'énigme de la personnalité), la capacité de celle-ci à se substituer à l'intersubjectivité restent débattues.

Deux modèles coexistent : un dispositif professionnel mis en œuvre par des agents spécialisés, qui prétend à la suprématie et un dispositif amateur auquel les directeurs de supermarché de la grande distribution restent attachés. On peut penser que si ce dernier résiste à l'impératif social de scientificité, c'est qu'il n'est pas qu'une survivance d'une forme désuète mais qu'il permet d'affronter l'énigme que pose toute personne. Face à la démarche des professionnels qui tendent à multiplier les outils techniques supposés combler un déficit cognitif, dire la vérité du candidat, les tenants du dispositif amateur tendent à privilégier l'entretien qui permet de déployer une sociologie des sens, de se le représenter en situation, dans son activité à venir. C'est en outre le moyen le plus assuré d'apprécier s'il est un être loyal, digne de confiance et la confiance n'est pas seulement cognitive (un problème d'informations, de savoirs) mais comporte toujours une part de risque. Elle est une forme d'engagement pour laquelle le face-à-face est jugé sans équivalent. 


\section{Références bibliographques :}

Amossy R. (1999), « L'ethos au carrefour des disciplines : rhétorique, pragmatique, sociologie des champs ", in Images de soi dans le discours, Lausanne, Delachaux et Niestlé.

Autor D.H. (2001), « Wiring the Labor Market », Journal of Economic Perspectives, Vol.15, N¹, Winter.

Cohen Y. (1993), « Aux origines de la direction du personnel aux Etats-Unis (1900-1918) », Personnel, №343, juill.

Eymard-Duvernay F., Marchal E. (1996), Façons de recruter. Le jugement des compétences sur le marché du travail, Paris, Métailié.

Fombronne J. (2001), Personnel et DRH. L'affirmation de la fonction Personnel dans les entreprises (France, 1830-1990), Paris, Vuibert.

Fondeur Y., Tuchszirer C. (2005), Internet et les intermédiaires du marché du travail, Rapport, IRES, Noisy-le-Grand, juin.

Granovetter M. (1974), Getting a Job, $2^{\text {nd }}$ ed., Chicago, University of Chicago Press, 1985.

Larson M.S. (1977), The Rise of Professionalism. A Sociological Analysis, Berkeley, California University Press.

Lochard Y., Ughetto P. (2004), « Candidat à un emploi : jeux et enjeux de la sollicitation ", Documents de travail de l'IRES, N04.11, décembre.

Lochard Y., Ughetto P. (2006), « Comment les directeurs de magasins recrutent. Critères et modalités de l'embauche ", Documents de travail de l'IRES, $\mathrm{N}^{\circ} 06.03$, juin.

Marchal E. (2005), « Compétence et graphologie : la mise en valeur de la personnalité ", in M.-C. Bureau, E. Marchal, Eds., Au risque de l'évaluation. Salariés et candidats à l'emploi soumis aux aléas du jugement, Villeneuve-d'Ascq, Presses universitaires du Septentrion.

Marchal E., Torny D. (2003), « Des petites aux grandes annonces : évolution du marché des offres d'emploi (1960-2000) », Travail et emploi, N95.

Peeters H., Charlier P. (1999), « Contribution à une théorie du dispositif », Hermès, $\mathrm{N}^{\circ} 25$.

Polanyi K. (1944), La grande transformation. Aux origines politiques et économiques de notre temps, trad. fr., Paris, Gallimard, coll. NRF/Bibliothèque des sciences humaines, 1983.

Rees A. (1966), « Information Networks in Labor Markets », American Economic Review, Vol.56, №1/2, March.

Simmel G. (1908), « Essai sur la sociologie des sens », trad. fr., reproduit in Sociologie et épistémologie, PUF, coll. Sociologies, 1981. 